\section{Tacrolimus is poorly tolerated by patients with primary sclerosing cholangitis}

In light of the promising results of a pilot study, Talwalkar et al. investigated whether or not tacrolimus is indeed safe and efficacious in patients with primary sclerosing cholangitis (PSC).

Sixteen PSC patients with serum alkaline phosphatase levels at least 1.5 times the upper limit of normal were enrolled in this open-label phase II trial. Participants received tacrolimus $0.05 \mathrm{mg} / \mathrm{kg}$ twice daily (target whole-blood concentration $3-7 \mathrm{ng} / \mathrm{ml}$ ) for 1 year. The tacrolimus dose was adjusted at least once in nine of the participants based on their whole-blood tacrolimus concentration, serum creatinine level or drug-related adverse events reported.

Drug-related adverse events were observed in 13 participants. There were eight participants who failed to complete the treatment: five withdrew owing to drug-related adverse effects, two failed to take the drug after enrolment and one died in an accident. A statistically and clinically significant reduction in the median serum alkaline phosphatase level (903 vs $483, P=0.0001$ ) was observed in the eight patients who completed treatment, and there was a statistically but not clinically significant reduction in the median aspartate aminotransferase level ( 88 vs $78, P=0.002)$. No statistically or clinically significant reductions in total bilirubin or albumin levels were observed.

Although these findings confirm that tacrolimus improves the serum alkaline phosphatase level in patients with PSC, the authors suggest that the clinical benefit of tacrolimus is limited due to poor patient tolerance.

Original article Talwalkar JA et al. (2007) Tacrolimus for the treatment of primary sclerosing cholangitis. Liver Int 27: 451-453

\section{Technique for early prediction of local response to RT in patients with HCC}

Radiation therapy (RT) has been shown to achieve reasonable local control of hepatocellular carcinoma (HCC). Liang and colleagues investigated whether dynamic contrastenhanced MRI (DCEMRI) data obtained in the second week of an RT regimen could predict treatment outcomes in 19 patients with HCC.
The patients included in this study (mean age 64 years, $84.2 \%$ men) had not responded to local therapy, and had not received any treatment for $\geq 1$ month before beginning RT. The tumor-targeted $\mathrm{RT}$ regime involved a total of 50 Gy administered in one 2 Gy daily fraction, 5 days per week, for 5 weeks. Patients underwent DCEMRI for the evaluation of change in signal intensity before RT, after 2 weeks' (20 Gy) RT, and 1 month after the end of the RT course (i.e. 9 weeks after commencement). The response of liver tumors was evaluated by conventional MRI.

DCEMRI initial enhancement slope (the steepest slope of the first rise on the timeintensity curve) and peak enhancement ratio ([maximum signal intensity-base signal intensity] $\div$ base signal intensity) measurements of the tumor at week 2 were significantly higher in patients who had good local control of HCC than in those who had poor control $(P=0.005$ and $P=0.017$, respectively). The same DCEMRI measurements of hepatic parenchyma were significantly higher at week 2 in patients who developed recurrence or metastasis than in those who did not (slope $P=0.017$, peak $P=0.033$ ).

The authors conclude that DCEMRI performed after 2 weeks' RT might help predict local responses to RT in patients with HCC.

Original article Liang PC et al. (2007) Dynamic MRI signals in the second week of radiotherapy relate to treatment outcomes of hepatocellular carcinoma: a preliminary result. Liver Int 27: 516-528

\section{IBS associated with non-appendicitis appendectomy}

Researchers in Taiwan have tested the hypothesis that patients who undergo appendectomy after misdiagnosis of appendicitis will have a higher incidence of IBS and worse psychological profiles and health-related quality of life scores than those correctly diagnosed as having acute appendicitis.

Lu and colleagues from Taipei Veterans General Hospital did a prospective, longitudinal study of 430 consecutive patients scheduled to undergo emergency appendectomy. Patients completed the Rome-II IBS questionnaire, the Hospital Anxiety and Depression Scale, and the ShortForm 36 survey after surgery. All removed appendices were analyzed pathologically and 